VOL. $24(1981), 27-36$.

\title{
FORCE DISTRIBUTION ON A SLENDER BODY CLOSE TO AN INTERFACE
}

\author{
J.R. BLAKE AND G,R, FULFORD
}

\begin{abstract}
The motion of a slender body parallel and very close to a flat interface which separates two immiscible liquids of differing density and viscosity is considered for very small Reynolds numbers. Approximate analytical expressions are obtained for the distribution of forces acting on the slender body. The limiting case of a rigid plane wall yields results obtained previously.
\end{abstract}

\section{Introduction}

Slender bodies moving in a viscous fluid, where the Reynolds number is very small, occur in the biological, engineering and industrial fields. In many cases the movements of these slender bodies are affected by the presence of an interface with another fluid or a boundary, either rigid or flexible. Examples include the locomotion of micro-organisms, extrusion of polymers, bubble flotation processes and the mechanics of colloidal suspensions.

Previously, many studies have concerned the motion of particles near a rigid plane wall boundary. In particular, Katz et al [4], using a line distribution of stokeslets, obtained approximate analytical expressions for the force distribution on an axisymmetric slender body oriented parallel to a plane wall and moving along one of its three principal axes. More recently however, some attention has been given to the more general problem of a particle moving in the presence of, or straddling the interface

Received 19 January 1981. 
between two immiscible fluids. An interface is a region (usually monomolecular) separating two fluids of different viscosities and densities. Lee et $a Z$ [5] and $O^{\prime} \mathrm{Nei} / \mathrm{l}$ and Ranger [7] have investigated the motion of a translating and rotating sphere in the presence of an interface which they both assume to be flat due to the action of surface tension and hydrostatic pressure. Clearly there is a need to examine the motions of other types of particles and in particular, the motion of a slender body near this type of boundary.

In this paper we extend the results of Katz et al [4] to that of a slender body moving close to a flat interface between two immiscible, incompressible, viscous fluids in the limit of zero Reynolds number. Expressions are presented for the force distributions on the slender body and the two limiting cases of a plane wall and a flat free surface are analysed in detail.

\section{Slender body theory}

Consider an axisymmetric slender body of length 22 and radius $r_{0}(x)$ (which is a smoothly varying function of distance along the axis of symmetry of the body). The body is moving with velocity $U$ in the lower of the two fluids $\left(x_{3}<0\right)$ (which has a dynamic viscosity $\mu_{1}$ and density $\rho_{1}^{*}$ ) at a distance $h$ from a flat interface (see Figure 1) where $r_{0} \ll h \ll z$. We assume that the interface is not surface active and consequently the interfacial tension $\gamma$ is constant.

The Green's function for a flat interface between two immiscible viscous fluids, derived by Adergoba and Blake [1], is

(1) $G_{i j}=\frac{1}{8 \pi \mu_{1}}\left[\frac{\delta_{i j}}{r}+\frac{r_{i} r_{j}}{r^{3}}+\left(\frac{1-\theta}{1+\theta} \delta_{j \alpha} \delta_{\alpha k}-\delta_{j 3} \delta_{3 k}\right)\left(\frac{\delta_{i k}}{R}+\frac{R_{i k} R_{k}}{R^{3}}\right)\right.$

$$
\begin{aligned}
& \left.+\frac{2 \theta}{1+\theta} h\left(\delta_{j \alpha} \delta_{\alpha k}-\delta_{j 3} \delta_{3 k}\right) \frac{\partial}{\partial R_{k}}\left(\frac{h R_{i}}{R^{3}}+\frac{\delta_{i 3}}{R}+\frac{R_{i} R_{3}}{R^{3}}\right]\right), \\
& (i, j, k=1 \ldots 3) \text { and }(\alpha=1 \ldots 2)\left(x_{3}<0\right)
\end{aligned}
$$

where $\theta=\mu_{2} / \mu_{1}$, the ratio of viscosities of the two fluids. The coordinate $r$ measures the distance from the point force (which is located 


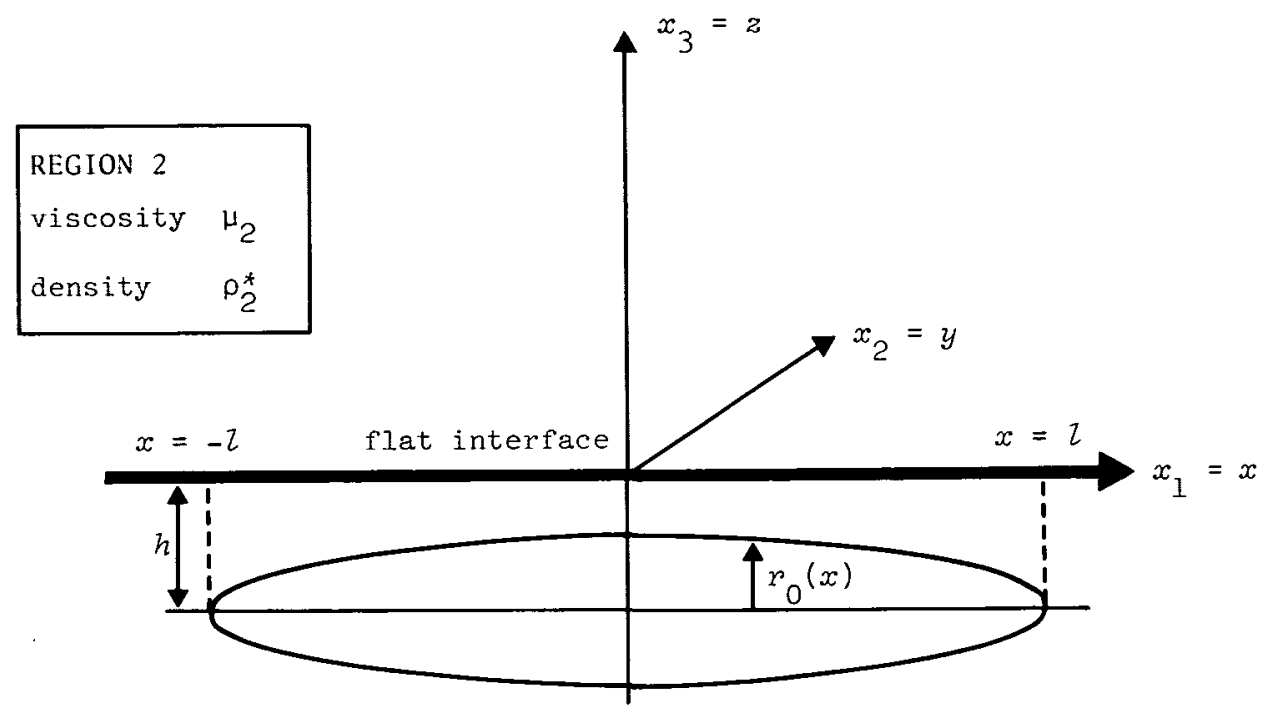

\begin{tabular}{lll}
\hline REGION & 1 & \\
viscosity & $\mu_{1}$ \\
density & $\rho_{1}^{*}$
\end{tabular}

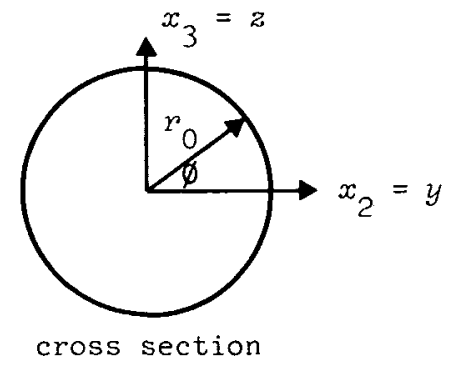

FIGURE 1. Slender body translating very close to and parallel to a flat interface which separates two fluids of different viscosities and densities. 
at $x_{3}=-h$ ) and $R$ measures the distance from the image point (located at $x_{3}=h$ ). By distributing these singularities along the centreline of the body we obtain the following integral representation for the velocity field of the fluid, $u(x)$ :

$$
u_{i}(x)=\int_{-2}^{2} G_{i j}(x, s) F_{j}(s) d s
$$

where the repeated suffix summation convention is used. Upon satisfying the no-slip boundary condition $u=U$ on the surface of the slender body, integral equations for the unknown force distributions $F(x)$ are obtained. In the separate cases of

(i) longitudinal motion (in the same direction as the axis of symmetry of the slender body) we take $F=\left(E_{1}, 0,0\right)$ and $U=\left(U_{1}, 0,0\right)$,

(ii) transverse motion (normal to the axis but parallel to the flat interface) we take $F=\left(0, F_{2}, 0\right)$ and $U=\left(0, U_{2}, 0\right)$, and

(iii) normal motion (normal to both the axis and the interface) we take $\mathrm{F}=\left(0,0, E_{3}\right)$ and $U=\left(0,0, U_{3}\right)$.

We note that in the more general circumstances, where no assumption is made on the relative magnitudes of $h$ and $l$, it proves necessary for longitudinal and normal motion of the slender body to take $F=\left(F_{1}, 0, F_{3}\right)$ (that is to include a distribution of stokelets oriented normal to the motion - see, for example, de Mestre and Russell [6]). Longitudinal motion is further complicated by a rotation of the slender body about a line through its centre parallel to the $x_{2}$ axis. Neither of these problems concern us for close approaches to the interface (that is, $r_{0} \ll h \ll l$ ).

The three integral equations in the cases of longitudinal, transverse and normal motion are, using polar coordinates $\left(r_{0}, \phi, x\right)$, 
(3) $8 \pi \mu_{1} U_{1}=\int_{-2}^{2} E_{1}(s)\left\{\left(\frac{1}{r}+\frac{(x-s)^{2}}{r^{3}}\right)+\left(\frac{1-\theta}{1+\theta}\right)\left(\frac{1}{R}+\frac{(x-s)^{2}}{R^{3}}\right)\right.$ $\left.+\frac{2 \theta h}{1+\theta}\left(r_{0} \sin \phi-h\right)\left\{\frac{1}{R^{3}}-\frac{3(x-s)^{2}}{R^{5}}\right)\right\} d s$,

(4) $8 \pi \mu_{1} U_{2}=\int_{-\tau}^{\tau} F_{2}(s)\left\{\left(\frac{1}{r}+\frac{r_{0}^{2} \cos ^{2} \phi}{r^{3}}\right)+\left(\frac{1-\theta}{1+\theta}\right)\left(\frac{1}{R}+\frac{r_{0}^{2} \cos ^{2} \phi}{R^{3}}\right)\right.$ $\left.+\frac{2 \theta h}{1+\theta}\left(r_{0} \sin \phi-h\right)\left(\frac{1}{R^{3}}-\frac{3 r_{0}^{2} \cos ^{2} \phi}{R^{5}}\right)\right\} d s$,

(5) $8 \pi \mu_{1} U_{3}=\int_{-2}^{l} F_{3}(s)\left\{\left(\frac{1}{r}+\frac{r_{0}^{2} \sin ^{2} \phi}{r^{3}}\right)-\left(\frac{1}{R}+\frac{\left(r_{0} \sin \phi-2 h\right)^{2}}{R^{3}}\right)\right.$

$$
\left.-\frac{2 \theta h}{1+\theta}\left(r_{0} \sin \phi-h\right)\left(\frac{1}{R^{3}}-\frac{3\left(r_{0} \sin \phi-2 h\right)^{2}}{R^{5}}\right)\right\} d s \text {, }
$$

where now $r^{2}=(x-s)^{2}+r_{0}^{2}$ and $R^{2}=(x-s)^{2}+r_{0}^{2}-4 h r_{0} \sin \phi+4 h^{2}$.

\section{Force distributions}

For close approaches of a slender body to the interface we take $r_{0} \ll h \ll l$ and expand equations (3)-(5), retaining only $O(1)$ terms. The resulting equations are solved by making asymptotic expansions of the force distributions $F_{i}$ in integral powers of $\left[\ln \left(2 l / r_{0}\right)\right]^{-1}$. This procedure is common in slender body analysis in low Reynolds number flow and details of the analysis may be found in Blake [3].

Expressions for the force distributions correct to $O\left(\ln \left(2 l / r_{0}\right)\right)^{-2}$ are

(6) $F_{1}=\frac{2 \pi \mu_{1} U_{1}}{\ln \left(2 l / r_{0}(x)\right)+S^{*}(x)-\frac{1}{2}-((\theta-1) /(\theta+1))\left(\ln (2 / h)+S^{*}(x)-\frac{1}{2}\right)}$,

(7) $\quad F_{2}=\frac{4 \pi \mu_{1} U_{2}}{\ln \left(2 L / r_{0}(x)\right)+S^{*}(x)+\frac{1}{2}-((\theta-1) /(\theta+1))\left(\ln (2 / h)+S^{*}(x)\right)-\left(\frac{1}{2} \theta /(\theta+1)\right)}$ and 


$$
F_{3}=\frac{4 \pi \mu_{1} U_{3}}{\ln \left(2 h / r_{0}(x)\right)-\frac{1}{2}-\left(\frac{1}{2} \theta /(\theta+1)\right)}
$$

where

$$
S^{*}(x)=\frac{1}{2} \ln \left(1-x^{2} / z^{2}\right)
$$

If the ratio of viscosities $\theta$ is greater than 1 then the magnitudes of the force distributions are greater than those for a slender body translating in an infinite fluid (see Katz et al [4]). When $\theta$ is close to zero the same applies for the normal force, however the longitudinal and transverse force distributions are smaller than the corresponding infinite fluid values.

In the limiting case $\theta \rightarrow \infty$ equations (6)-(8) reduce to the Katz et al [4] results for a plane wall boundary (see Figure 2)

$$
F_{1}=\frac{2 \pi \mu_{1} U_{1}}{\ln \left(2 h / r_{0}^{2}\right)} \text {, }
$$

$$
F_{2}=\frac{4 \pi \mu_{1} U_{2}}{\ln \left(2 h / r_{0}\right)} \text {, }
$$

$$
F_{3}=\frac{4 \pi \mu_{1} U_{3}}{\ln \left(2 h / r_{0}\right)-1} \text {. }
$$

The closer the slender body to the boundary the greater is the magnitude of force acting on the body. In this case there is no dependence of the force distribution on axial distance $x$.

Another important limiting case is that of $\theta \rightarrow 0$ which corresponds to a boundary which is a flat free surface. The force distributions in this case are (see also Figure 2)

$$
\begin{aligned}
& E_{1}=\frac{2 \pi \mu_{1} U_{1}}{\ln \left[2 l^{2} / r_{0} h\right]+2 S^{*}(x)-1}, \\
& E_{2}=\frac{4 \pi \mu_{1} U_{2}}{\ln \left[2 l^{2} / r_{0} h\right]+2 S^{*}(x)+\frac{1}{2}}
\end{aligned}
$$

and 


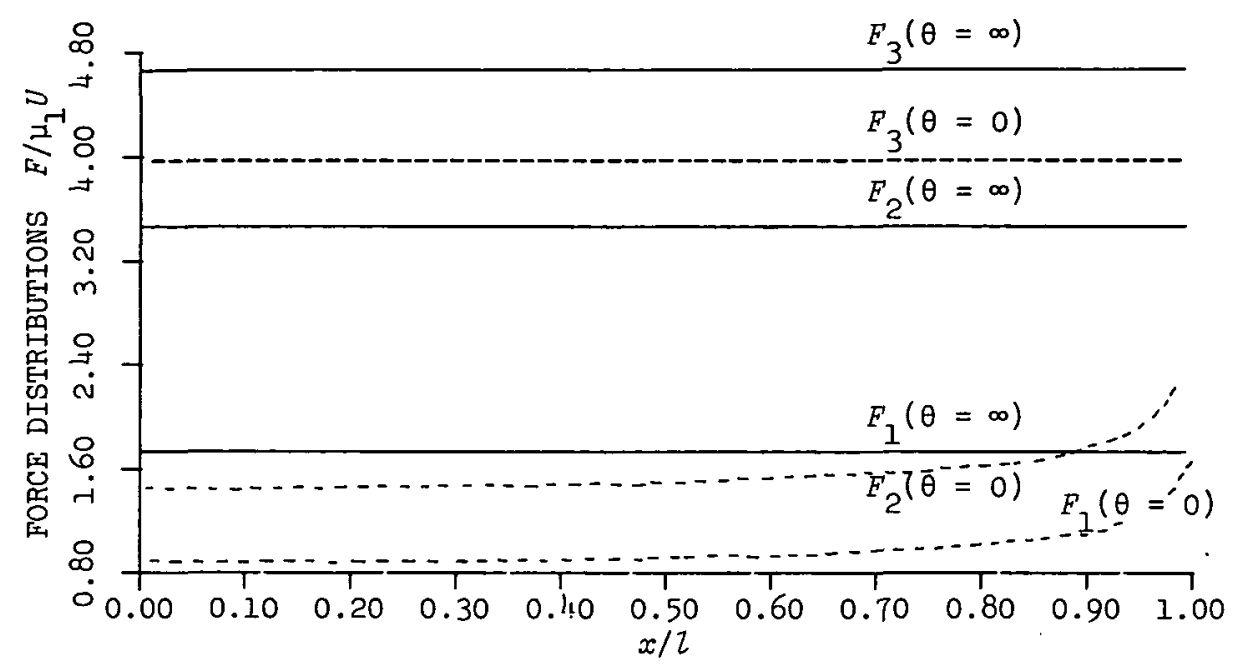

FIGURE 2. The force distributions on a slender cylinder $\left(R_{0} / 2=.005\right)$ which is translating close to $(h / 2=.1)$

(i) a plane rigid wall (solid lines), and (ii) a flat free surface (broken lines) given by equations (10)-(15) in text. Since the distributions are symmetric about the $x_{3}$-axis only the right half is plotted.

$$
F_{3}=\frac{4 \pi \mu_{1} U_{3}}{\ln \left[2 h / r_{0}\right]-\frac{1}{2}} .
$$

For motion normal to the free surface, the magnitude of the force distribution increases as the slender body moves closer to the free surface while for longitudinal and transverse motion it decreases.

It is important to realise that the $\theta=1$ case does not correspond to motion in an infinite fluid since although the viscosities of the two fluids are the same, their densities are different and there is an implied distribution of normal stress acting on the interface which causes the interface to remain flat. 


\section{Limitations}

In the analysis used to derive (6)-(8) we had assumed $r_{0} \ll h \ll 2$. Thus our solution is only valid when the slender body approaches sufficiently close to the interface (that is, well within one body length but still several radii away). The error involved in making the asymptotic expansions of the force distributions are of the order $\left[\ln \left(22 / r_{0}\right)\right]^{-3}$ which is compariable to $O\left(r_{0} / l\right)$ when $r_{0} / 2 \doteqdot .005$. The asymptotic results are invalid near the endpoints of the slender body for bodies where $\lim _{x \rightarrow \pm l} \frac{r_{0}(x)}{x^{2}-l^{2}}$ does not exist (for example, a circular cylinder). In these circumstances, end effects should be modelled by placing a distribution of stokeslets over the surfaces of the ends of the body.

For such close approaches to the interface, it is realised that the shape of the interface will be distorted from its assumed flat shape. Aderogba and Blake [2] obtained expressions for a first order approximation of the interface elevation $\zeta$ in the special cases where the flattening out effect is dominated by

(i) surface tension $(\gamma)$, and

(ii) hydrostatic pressure $\left(\rho_{1}^{*}-\rho_{2}^{*}\right) g \zeta$ where $g$ is the acceleration due to gravity.

By scaling with respect to the distance from the interface $h$ and taking the total force on the body as $2 F Z$ (since $F$ is constant along most of the body except near the end points) one obtains the conditions corresponding to $(i)$ and (ii) respectively

$$
\frac{\mu U}{\gamma}=0(h / 2)
$$

and

$$
\frac{\mu U}{\left(\rho_{1}^{*}-\rho_{2}^{*}\right) g h^{2}}=o(h / \tau)
$$

Thus the flat interface approximation is justified when there is a large interfacial tension acting on the fluids or a large density difference between the two fluids. 


\section{Summary}

Using distributions of stokeslets along the centreline of a slender body, which is translating parallel and very close to a flat interface between two immiscible fluids, we were able to obtain expressions for the force distributions on the slender body. It was shown that the force distributions (which are approximately constant along most of the slender body, except near the endpoints) in the limiting case of a rigid plane boundary, are greater than the equivalent results for an infinite fluid. For a flat free surface boundary the force distributions were found to be greater for motion normal to the free surface but less for tangential motion parallel to the free surface.

It is expected that the results obtained in this paper will have important consequences regarding our understanding of the motion of particles near different types of fluid-fluid interfaces.

\section{References}

[1] K. Aderogba and J.R. Blake, "Action of a force near the planar surface between two semi-infinite immiscible liquids at very low Reynolds numbers", Bulz. Austral. Math. Soc. 18.(1978), 345-356.

[2] K. Aderogba and J.R. Blake, "Action of a force near the planar surface between two semi-infinite immiscible liquids at very low Reynolds numbers: Addendum", Buzl. Austral. Math. Soc. 19 (1978), 309-318.

[3] J.R. Blake, "Singularities of viscous flow. Part II: Applications to slender body theory", J. Engrg. Math. 8 (1974), 113-124.

[4] D.F. Katz, J.R. Blake and S.L. Paveri-Fontana, "On the movement of slender bodies near plane boundaries at low Reynolds number", $J$. Fluid Mech. 72 (1975), 529-540.

[5] S.H. Lee, R.S. Chadwick and L.G. Leal, "Motion of a sphere in the presence of a plane interface. Part I: An approximate solution by generalisation of the method of Lorentz", J. Eluid Mech. 93 (1979), 705-726. 
[6] N.J. de Mestre W.B. Russell, "Low-Reynolds-number translation of a slender cylinder near a plane wall", J. Engrg. Math. 9 (1975), $81-91$.

[7] M.E. O'Neill and K.B. Ranger, "On the rotation of a rotlet or sphere in the presence of an interface", Internat. J. Multiphase Flow 5 (1979), 143-148.

Department of Mathematics,

University of Wollongong,

Woll longong,

New South Wales 2500,

Australia. 